\title{
Zur (Fach-)Spezifität von Unterrichtsqualität im Fach Sport
}

\author{
Alfred Richartz $\cdot$ Kathrin Kohake
}

Eingegangen: 21. Dezember 2020 / Überarbeitet: 27. April 2021 / Angenommen: 29. April 2021 / Online publiziert: 8. Juni 2021

(C) Der/die Autor(en) 2021

Zusammenfassung In der empirischen Unterrichtsforschung wird Unterrichtqualität anhand von Merkmalen beurteilt, die sich auf die Wirksamkeit von Unterricht beziehen. Auch der Sportunterricht wird zunehmend unter der Perspektive der Unterrichtsqualität analysiert. Dabei spielen generische Qualitätsdimensionen wie Klassen- und Zeitmanagement und sozio-emotionale Unterstützung der Schüler*innen eine wesentliche Rolle. Fachtypische Lerngelegenheiten des Sportunterrichts weisen Überschneidungsbereiche jedoch auch maßgebliche Unterschiede zu anderen Fächern auf: Schüler*innen sollen einerseits fachspezifisches Wissen und Können erwerben, um ihre Teilnahme an der Sport- und Bewegungskultur zu fördern. Andererseits sollen Schüler*innen aber wie in anderen Fächern Zusammenhänge verstehen und kritische Bewertungen vornehmen, um ihr Sporttreiben selbstbestimmt und verantwortlich zu gestalten. Die Qualität der Lernunterstützung im Sport muss sich daher auf drei Bereiche beziehen: auf motorisches Lernen, auf spieltaktisches Lernen und auf verständnis- und bewertungsbezogenes Lernen. Der Beitrag zeigt, dass generische Qualitätsmerkmale ergänzt werden sollten um Merkmale effektiver Unterstützung in den beiden sportspezifischen Lernfeldern. Hierzu zählen eine für das Bewegungslernen geeignete Aufmerksamkeitslenkung, spezifische Feedbacktechniken, sinnvolle didaktische Reduktion sowie ausreichende Lernzeit für die beim motorischen und taktischen Lernen erforderlichen Prozeduralisierungsprozesse.

Originalität: Die Autor/-innen geben an, den Beitrag an keiner anderen Stelle veröffentlicht oder zur Veröffentlichung eingereicht zu haben.

\footnotetext{
Alfred Richartz $\cdot$ Kathrin Kohake $(\bowtie)$

Institut für Bewegungswissenschaft, Arbeitsbereich Bewegungs- und Sportpädagogik, Universität

Hamburg, Turmweg 2, 20148 Hamburg, Deutschland

E-Mail: kathrin.kohake@uni-hamburg.de

Alfred Richartz

E-Mail: alfred.richartz@uni-hamburg.de
} 
Schlüsselwörter Bewegungslernen · Instruktionsqualität · Kognitive Aktivierung · Feedback

\title{
(Subject-)specificity of teaching quality in physical education
}

\begin{abstract}
In educational research, teaching quality is assessed based on aspects related to teaching effectiveness. This, too, increasingly applies to Physical Education. Current studies emphasize generic quality dimensions such as classroom management and emotional support. Subject-typical learning opportunities in Physical Education, however, show overlapping areas but also significant differences to other subjects: On the one hand, students should acquire subject-specific knowledge and skills to promote their participation in leisure time physical activity. On the other hand, as in other subjects, students should understand conditions and structures of sports and make critical assessments to shape their physical activity in a self-determined and responsible way. Effective teaching in Physical Education must therefore relate to three areas: motor learning, game tactical learning, and deep understanding of concepts. The article shows that generic quality dimensions should be supplemented by dimensions of instructional support in both of the sport-specific learning areas. These include attention guidance suitable for motor learning, specific feedback techniques, effective didactic reduction as well as adequate pacing for the proceduralisation processes required in motor and tactical learning.
\end{abstract}

Keywords Motor learning · Instructional quality · Teaching for understanding · Feedback

\section{Die Diskussion der Fachspezifität von Unterrichtsqualität in der Sportdidaktik}

Aussagen über die Qualität von Unterricht implizieren Bewertungen, die auf unterschiedlichen Gütemaßstäben beruhen können. Die empirische Unterrichtsforschung hat bei der Entwicklung von fachübergreifenden Dimensionen der Unterrichtsqualität den Maßstab der Wirksamkeit zugrunde gelegt. Danach weist Unterricht dann eine hohe Qualität auf, wenn er ,lernwirksam“ ist (Helmke 2010, S. 20) und damit wahrscheinlich macht, dass Schüler*innen die beabsichtigten Lern- und Entwicklungsziele erreichen (Praetorius et al. 2020). Dieses Paradigma setzt voraus, dass die fachlichen Unterrichtsziele in einer Weise definiert sind, die ihre empirische Untersuchung gestattet.

Die einflussreichsten Strömungen in der deutschen Sportdidaktik standen diesem Grundverständnis bis weit in die 2000er Jahre mit Skepsis oder gar Ablehnung gegenüber, wie besonders die Diskussion um die Einführung von Bildungsstandards zeigt. Als schwerwiegendster Einwand wurde angeführt, dass Kernaspekte der Bildungsbedeutung von Sportunterricht nicht auf angemessene Weise messbar seien. Dabei wurde einerseits auf die grundsätzliche Reduktionsgefahr bei Operationalisierungen von fachlichen Bildungszielen hingewiesen (Gogoll und Kurz 2013; Scherler 2006) und andererseits der Stellenwert überfachlicher Ziele des Sportun- 
terrichts betont. Er soll beispielsweise die Entwicklung allgemeiner personaler und sozialer Kompetenzen und Haltungen fördern (Grupe et al. 2004), auf eine ganzheitliche Entwicklungsförderung gerichtet sein (Kurz 2008) sowie reflexive ästhetische Erfahrungen ermöglichen (Laging 2015). Zusätzlich soll Sportunterricht einen Ausgleich für die kognitive Ausrichtung des Schulalltags bieten und zur gesundheitlichen Entwicklung beitragen. Solche fachübergreifenden und weitgespannten Ziele werden mit Nachdruck auch von außen an das Fach herangetragen - vom schulischen Nahumfeld (Schüler*innen, Schulleitungen, vgl. Sprint-Studie: Deutscher Sportbund 2006), aber auch von bildungspolitischen Akteuren (Aschebrock 2013).

Das Kernkonzept des Effective-Education-Paradigmas war also in den wichtigsten sportdidaktischen Positionen weder einheimisch noch leicht anschlussfähig. Vor diesem Hintergrund sind die Ergebnisse der Literaturanalyse von Herrmann und Gerlach (2020) bemerkenswert. Zunächst überrascht, wie breit die Qualitätsdimensionen der empirischen Unterrichtsforschung in der sportdidaktischen Literatur aufgenommen wurden. Weiterhin fällt auf, dass nicht fachspezifische, sondern fachübergreifende Dimensionen wie Klassenführung und sozio-emotionale Unterstützung in den empirischen Studien zum Sportunterricht eine dominierende Rolle einnehmen (Herrmann und Gerlach 2020, S. 370 ff.). Die Überprüfung von Zusammenhängen zwischen diesen generischen Qualitätsdimensionen und fachlichen Outcomes im Sportkontext steht noch weitgehend aus. Vorliegende Studien zeigen jedoch das Potenzial dieser Übertragungen: Validität und Reliabilität der Unterrichtsqualitätsmerkmale konnten sowohl für Fragebogen- als auch für Beobachtungsstudien auf zufriedenstellendem Niveau gezeigt werden (z. B. Heemsoth und Krieger 2018; Richartz und Zoller 2011).

\section{Eigene Positionierung}

Praetorius und Charalambous (2018) haben zwölf Instrumente zur Erhebung der Unterrichtsqualität untersucht und in einem aufwändigen Syntheseprozess zu einem Rahmenmodell mit sieben Dimensionen zusammengeführt. Dabei heben sie als besonders herausfordernd die Entwicklung der kategorialen Ordnung hervor (ebd., S. 544 ff.). Denn ähnlich benannte Qualitätsdimensionen können in verschiedenen Instrumenten unterschiedliche Konstrukte bezeichnen, Teilaspekte (Subdimensionen) können in anderen Kategorisierungen über verschiedene Dimensionen verteilt sein u.ä. m. Überträgt man Dimensionen und Subdimensionen auf andere Kontexte (z.B. Altersstufen) oder andere Fächer, in unserem Fall Sport, entstehen ähnliche Probleme. Begriffe, die Tiefenstrukturen des Unterrichts kennzeichnen, müssen dann kontextspezifisch angemessen operationalisiert werden (Klieme 2006). Entscheidend ist, ob trotz solcher Anpassungen die Konstruktäquivalenz gewahrt bleibt.

Praetorius und Charalambous (2018) unterscheiden in ihrem Framework zwischen verschiedenen Graden erwartbarer Fachspezifität von Qualitätsdimensionen und Subdimensionen. So werden Klassen- und Zeitmanagement, sozio-emotionale Unterstützung sowie die laufende Aufmerksamkeit der Lehrkraft für Lernprobleme als vorwiegend überfachlich erwartet. Die Auswahl und Präsentation der Inhalte und die Evaluation des Lernfortschritts dagegen werden als fachspezifisch gekennzeich- 
net, die meisten anderen jedoch als hybrid - also gleichermaßen mit einem überfachlichen und einem fachlichen Anteil versehen. Die Literaturanalyse von Herrmann und Gerlach (2020) zeigt eine hohe Passung zwischen dem Syntheseframework und den Qualitätsmerkmalen, die in der Sportdidaktik thematisiert wurden. Dies betrifft insbesondere die generischen Dimensionen Klassen- und Zeitmanagement, sozio-emotionale Unterstützung sowie Unterstützung des Lernens aller Schüler*innen (Praetorius et al. 2020, S. 417ff.). Die hohe Anzahl einschlägiger Beiträge und die Ausdifferenzierung der Subdimensionen zeigt die Bedeutung, die diesen Qualitätsmerkmalen im Fach Sport zugeschrieben wird und der wir uns ausdrücklich anschließen. Im Folgenden konzentrieren wir uns auf die Frage, ob die Besonderheiten des fachlichen Lernens im Sport zusätzliche Qualitätsmerkmale wünschenswert machen oder besondere Schwierigkeiten für die Wahrung der Konstruktäquivalenz aufwerfen.

Die fachtypischen Lerngelegenheiten des Sportunterrichts ergeben sich aus dem Auftrag des Schulsports. Er wird seit einiger Zeit in der Formel eines „Doppelauftrages“ zusammengefasst (Kurz 2008; Gogoll und Kurz 2013). In einer ersten Zieldimension soll Sportunterricht Wissen und Können vermitteln, das die Schüler*innen befähigt, an der außerschulischen Sport- und Bewegungskultur teilzuhaben. Dazu ist ein grundlegendes Maß an körperlicher Fitness, an Bewegungskönnen und sportbezogenem Wissen erforderlich. Gogoll (2013) hat dies treffend als ,operative Handlungsfähigkeit“ bezeichnet. Wenn wir die Herbeiführung physiologischer Veränderungen (,Fitness“) hier unbeachtet lassen, sollten Unterrichtsanteile, die auf diese Zieldimension gerichtet sind, das Erlernen von Bewegungsfertigkeiten und die Fähigkeit zum Mitspielen in vielfältigen Bewegungsspielen fördern. Die Fähigkeit zum Mitspielen erfordert über die Beherrschung von Bewegungsfertigkeiten hinaus ihren zielgerichteten taktischen Einsatz: Spielkompetenz erfordert somit das Erlernen spezifischer Wahrnehmungs- und Entscheidungsvorgänge.

Die zweite Dimension des Doppelauftrages wird in der Literatur unterschiedlich akzentuiert (Kurz 2008). Gogoll (2013) prägt dafür den Begriff einer ,,reflexiven Handlungsfähigkeit“: Schüler*innen sollen in der Lage sein, ihr Sporttreiben zu analysieren und zu bewerten, um es selbstbestimmt und verantwortlich gestalten zu können - der Auftrag zielt also auf die Perspektive der Mündigkeit im Sporttreiben (Gogoll und Kurz 2013). Die damit verbundenen Lerngelegenheiten thematisieren das Verstehen innerer Zusammenhänge, kritische Bewertungen, eigenständiges Planen und Entscheiden.

Diese grobe Annäherung legt nahe, dass die Qualität der Lernunterstützung im Sportunterricht sich auf drei verschieden strukturierte Lernvorgänge beziehen muss: 1) Bewegungslernen und 2) spieltaktisches Lernen im Bereich der operativen Handlungsfähigkeit und 3) verständnis- und bewertungsbezogenes Lernen im Bereich der reflexiven Handlungsfähigkeit. Je nach Stundenschwerpunkt oder Unterrichtsphase können diese Lernprozesse in verschiedenen Anteilen vorkommen oder auch simultan in Aufgaben vernetzt sein (Pfitzner 2018). Den geringsten Überschneidungsbereich mit fachtypischen Lernvorgängen in anderen Fächern dürfte das Bewegungslernen besitzen, während beim reflexiv orientierten Lernen die Prozesse des Analysierens, Verstehens und Planens wie in anderen Fächern strukturiert sein dürften. Vordringlich ist deshalb zu prüfen, ob die Sportmotorik- und Sportspielforschung 
Hinweise liefern auf einen fachspezifischen Ergänzungsbedarf bisher unberücksichtigter Qualitätsdimensionen oder Subdimensionen. Dies kann hier natürlich nur in stark verkürzter Form versucht werden.

In der Motorikforschung werden Bewegungssteuerung und Bewegungslernen in kognitiven Wahrnehmungs-, Entscheidungs- und Steuerungsarchitekturen beschrieben (Hossner et al. 2013). Zum Beispiel werden in ideomotorischen Ansätzen interne Modelle postuliert, die Voreinstellungen darüber enthalten, mittels welcher motorischer Kommandos welche Effekte in der Umwelt erzeugt werden können (Prädiktormodell). Diese Modelle arbeiten prinzipiell implizit, lediglich die Effekte der Steuerungsabläufe (Bewegungsausführung, Situationsveränderung) sind bewusstseinsfähig. Veränderungen der modellinternen Voreinstellungen - also Lernen - findet über durch dauerhaft internalisierte Neueinstellungen der Steuerungsabläufe statt. Dabei spielen Übungsprozesse die wesentliche Rolle, also Versuche, die angezielten Effekte hervorzurufen und die interne, wiederum implizite Verarbeitung der rücklaufenden Ergebnisinformationen. Im Syntheseframework ist mit der Dimension „Unterstützung des Übens“ ein geeigneter Ort für diese sportunterrichtstypische Form des Lernens zu finden. Die Liste der zugehörigen Subdimensionen legt allerdings eine Erweiterung nah. Im Bewegungslernen haben Übungsprozesse nämlich nicht erst bei Konsolidierung des Gelernten, sondern bereits für das Neulernen eine zentrale Funktion. Die Eigenlogik des Bewegungslernens bedingt Besonderheiten effektiver Lernunterstützung. Während es in anderen Fächern nützlich ist, wenn Lernenden Prozeduren bewusst gemacht werden (,Prozedur beschreiben“; vgl. Praetorius et al. 2020, S. 419), sollte die Aufmerksamkeit beim Bewegungslernen gerade von (vermeintlichen) Steuerungsprozessen weg und stattdessen auf den angezielten Effekt gelenkt werden (Scherer und Bietz 2013). So ist die Aufmerksamkeitslenkung auf das Bewegungsergebnis (ein Ball landet im Korb) lernwirksamer als die Aufmerksamkeitslenkung darauf, wie die Bewegung funktioniert (Ablauf der Handbewegung, externaler statt internaler Fokus; Wulf 2013). Diese Besonderheit stellt ein spezifisches instruktionales Qualitätsmerkmal dar.

Auch prozessbegleitendes Feedback hat eine hohe Bedeutung beim Bewegungslernen. Eine fachspezifische Besonderheit ist dabei der oft hohe nonverbale Anteil (Rhythmisierungen, Auslösesignale für Bewegungen, taktiles Feedback). Ein weiterer wichtiger Aspekt ist die passgenaue Begleitung eines Lernenden über mehrere Stufen zum Zielzustand. Solche Prozesse werden als „Scaffolding“ auch in anderen Fächern beschrieben (van de Pol et al. 2010). Dieses Tiefenkonzept wird im Sport oft durch fachspezifische Hilfen realisiert: Einschränkungen des Bewegungsraums, physische Hilfen, Auslösesignale, Aufmerksamkeitslenkung. Diese fachspezifischen Qualitätsmerkmale sind aus unserer Sicht für lernwirksamen Sportunterricht hoch relevant.

Für den Lernbereich des Mitspielens, z.B. in den Ballspielen, tritt neben dem erforderlichen Bewegungskönnen als zentrale Aufgabe hinzu, taktische Entscheidungen zu treffen. Die typische Aufgabenstruktur wird in der Forschung als Auswahlentscheidung unter IF-THEN-Regeln modelliert (Raab et al. 2009). Die zugrundeliegende Regelstruktur ist deklarativ zugänglich und häufig wenig komplex. Sie kann für Zielschussspiele z. B. lauten: „Schaust du frei aufs Tor, dann schieß! Ist der Weg blockiert, dann passe zum Mitspieler!“ Die spezifische Schwierigkeit 
der Aufgabenlösung wird vor allem erzeugt durch hohen Zeitdruck: Aktionen von Gegenspieler*innen ändern die Entscheidungslage in Sekundenbruchteilen. Deklaratives Problemlösen ist deshalb ungeeignet - es wird eine prozeduralisierte und damit schnellere Aufgabenlösung erzwungen. Beim Anfängerlernen kommt hinzu, dass vorhandene dysfunktionale automatisierte Handlungsmuster inhibiert werden müssen. So laufen Kinder gern zum Ballbesitzenden statt sich freizulaufen. Die erforderliche Lernsequenz benötigt einen hohen Prozeduralisierungsaufwand - der auf der Sichtstruktur des Unterrichts natürlich nicht als ödes Üben materialisiert sein muss (Mitchell et al. 2013; Roth und Kröger 2015). Es liegen verschiedene Instruktionsstrategien für spieltaktisches Lernen vor: entdeckendes Lernen, gelenktes implizites Lernen, deklaratives Lernen mit anschließender Automatisierung (Raab et al. 2009). Welche Vermittlungsmodelle besonders effektiv sind, hängt offenbar auch von situativen Faktoren ab (Komplexität der Aufgabe, Kompetenz der Lerner; Raab 2007).

Alle Elemente dieser Lehr-Lern-Sequenz sind im Syntheseframework in den Dimensionen „Unterstützung des Übens“ und ,Auswahl und Thematisierung von Inhalten“ problemlos zu verorten, vor allem wenn die vorstehend vorgeschlagenen Ergänzungen berücksichtigt werden. Im Vergleich zu Lernsequenzen anderer Fächer ist aber auf einen bescheidenen deklarativ-verstehenden Aufwand und einen hohen Aufwand für die Prozeduralisierung hinzuweisen. Deshalb hat unseres Erachtens eine auf diese Lernsequenz angepasste Auswahl und Strukturierung von Fachmethoden eine wichtige Bedeutung. Dazu gehört insbesondere, wie für naturwissenschaftliche Fächer (Praetorius et al. 2020, S. 417), eine angemessene didaktische Reduktion.

In der allgemeinen Unterrichtsforschung spielt die Dimension der kognitiven Aktivierung eine herausragende Rolle (Stürmer und Fauth 2019). Wie Herrmann und Gerlach (2020, S. 370) hervorheben, ist es besonders herausfordernd, diese Dimension für den Sportunterricht fruchtbar zu machen. Der Kern des Konzepts besteht in der Unterscheidung zweier Ebenen von kognitiven Prozessen und damit Lernvorgängen. Eine untere Ebene ist gekennzeichnet durch bloßes Memorieren und Reproduzieren von unverbundenen deklarativen Wissenselementen (,pieces of information“, Darling-Hammond 2008, S. 1) sowie der Anwendung basaler, automatisierter Fertigkeiten. Bei den Vorgängen auf dieser Ebene handelt es sich auch um kognitive Operationen, aber es sind nicht solche, die durch die Aktivierung angesprochen werden sollen. Auf einer (je nachdem) höheren (,,higher-order thinking“, Lewis und Smith 1993) oder tieferen Ebene (,vertieftes Verstehen“) finden dagegen vertiefte, ,,anspruchsvollere“ (Stürmer und Fauth 2019, S. 8) und verständnisfördernde Operationen statt: Neue Wissenselemente werden mit bestehenden verknüpft, Präkonzepte werden aktiviert und durch Auseinandersetzung mit dem Lerngegenstand umgearbeitet, konzeptuelle Wissensstrukturen ermöglichen, Zusammenhänge zu erfassen, Transfer zu leisten und neue Problem zu lösen statt bekannte Lösungen zu repetieren (Donovan und Bransford 2005; Darling-Hammond 2008; Stürmer und Fauth 2019). Die Gestaltung von Lernumgebungen und Lehrverhalten entscheidet darüber, ob Lernprozesse eher auf ,niederer“ oder ,,aktivierter“ Ebene angestoßen werden z.B. Auswendiglernen oder problemlösendes Lernen.

Dieses Konzept eines auf vertieftes Verstehen orientierten Lernens lässt sich auf viele Lerngegenstände übertragen - im Sportunterricht kommen bei Wahrung der 
Konstruktäquivalenz jedoch vor allem die Lerngelegenheiten im Zielbereich der reflexiven Handlungsfähigkeit in Frage. Hier kann man die Thematisierung von Präkonzepten erwarten, problemlösendes Lernen und die Anwendung von Fähigkeiten auf neue komplexe, soziale und wertbezogene Probleme.

Auch im Bereich der operativen Handlungsfähigkeit werden durchgängig kognitive Lernvorgänge benötigt - aber solche auf Ebene von ,,higher-order thinking“ sind weit weniger wichtig oder können mitunter sogar hinderlich sein. Bereits beim taktischen Lernen handelt es sich um eine geringe Zahl von guten Lösungen und festliegenden Entscheidungsregeln. Diese können durch problemlösendes Lernen entdeckt werden, die Entwicklung taktischen Könnens geschieht jedoch, wie zu vor beschrieben, vor allem durch Prozeduralisierung. Noch weniger angebracht erscheint kognitive Aktivierung in der Förderung des Bewegungskönnens, da hier vor allem implizite Lernprozesse im Mittelpunkt stehen. Dennoch macht es wenig Sinn, zwischen Kognition einerseits und Motorik andererseits zu unterscheiden, weil motorische Steuerung selbst vor allem als Zusammenspiel von Planungs-, Wahrnehmungs- und Entscheidungsprozessen verstanden werden muss (Wolpert und Landy 2012).

Reale Lernumgebungen kombinieren stets kognitive Aktivitäten auf „basaler“ und ,,vertiefter“ Ebene - ,higher-order thinking“ setzt „,basic skills“ voraus (Lewis und Smith 1993). Es geht also, gerade im Sportunterricht, nicht um eine polare Gegenüberstellung. Deshalb sind die aktuellen Ansätze einer konstruktäquivalenten Übertragung von kognitiver Aktivierung in den Sportunterricht sehr wertvoll (z. B. Hapke und Waigel 2019). Sie berühren aber nur einen Teilbereich der fachspezifischen Lerngelegenheiten und können deshalb nicht so zentrale Bedeutung für die Qualität der Lernunterstützung haben wie in anderen Fächern.

Aus unserer Sicht haben generische Qualitätsdimensionen, wie sie im Syntheseframework aufgeführt werden, für die Unterrichtsqualität im Sport eine entscheidende Bedeutung. Es erscheint uns jedoch sehr wünschenswert, weitere Qualitätsmerkmale zu berücksichtigen, die die Erfordernisse spezifischer Lernvorgänge im Sport adressieren. Dazu gehören eine geeignete Aufmerksamkeitslenkung auf (erwartete) Effekte statt auf Steuerungsprozesse beim Bewegungslernen, damit verbunden ein spezifisches Repertoire an Feedbacktechniken (Scaffolding, nonverbale oder taktile Impulse), eine auf die Lernsequenz abgestimmte angemessene didaktische Reduktion sowie angemessene Prozeduralisierungsgelegenheiten.

Funding Open Access funding enabled and organized by Projekt DEAL.

Open Access Dieser Artikel wird unter der Creative Commons Namensnennung 4.0 International Lizenz veröffentlicht, welche die Nutzung, Vervielfältigung, Bearbeitung, Verbreitung und Wiedergabe in jeglichem Medium und Format erlaubt, sofern Sie den/die ursprünglichen Autor(en) und die Quelle ordnungsgemäß nennen, einen Link zur Creative Commons Lizenz beifügen und angeben, ob Änderungen vorgenommen wurden.

Die in diesem Artikel enthaltenen Bilder und sonstiges Drittmaterial unterliegen ebenfalls der genannten Creative Commons Lizenz, sofern sich aus der Abbildungslegende nichts anderes ergibt. Sofern das betreffende Material nicht unter der genannten Creative Commons Lizenz steht und die betreffende Handlung nicht nach gesetzlichen Vorschriften erlaubt ist, ist für die oben aufgeführten Weiterverwendungen des Materials die Einwilligung des jeweiligen Rechteinhabers einzuholen. 
Weitere Details zur Lizenz entnehmen Sie bitte der Lizenzinformation auf http://creativecommons.org/ licenses/by/4.0/deed.de.

Interessenkonflikt K. Kohake und A. Richartz geben an, dass kein Interessenkonflikt besteht.

\section{Literatur}

Aschebrock, H. (2013). Vom Sportartenprogramm zur Kompetenzorientierung - zum Wandel curricularer Leitideen. In H. Aschebrock \& G. Stibbe (Hrsg.), Didaktische Konzepte für den Schulsport (S. 53-78). Aachen: Meyer \& Meyer.

Darling-Hammond, L. (2008). Powerful learning: what we know about teaching for understanding. San Francisco: John Wiley.

Deutscher Sportbund (2006). DSB-Sprint-Studie. Aachen: Meyer \& Meyer.

Donovan, S., \& Bransford, J. (2005). How students learn. History, mathematics, and science in the classroom. Washington: National Academies Press.

Gogoll, A. (2013). Sport- und bewegungskulturelle Kompetenz. Zur Begründung und Modellierung eines Teils handlungsbezogener Bildung im Fach Sport. Zeitschrift für Sportpädagogische Forschung, 1(2), $5-24$.

Gogoll, A., \& Kurz, D. (2013). Kompetenzorientierter Sportunterricht - das Ende der Bildung? In H. Aschebrock \& G. Stibbe (Hrsg.), Didaktische Konzepte für den Schulsport (S. 79-97). Aachen: Meyer \& Meyer.

Grupe, O., Kofink, H., \& Krüger, M. (2004). Gegen die Verkürzung von Bildung auf Bildungsstandards im Schulsport. Sportwissenschaft, 34(4), 484-495.

Hapke, J., \& Waigel, S. (2019). „Sporttreiben mit Köpfchen“ - Kognitive Aktivierung im Sportunterricht. In A. Gawatz \& K. Stürmer (Hrsg.), Kognitive Aktivierung im Unterricht. Befunde der Bildungsforschung und fachspezifische Zugänge (S. 148-161). Braunschweig: Westermann.

Heemsoth, T., \& Krieger, C. (2018). Perspektiven auf Sportunterricht. Unterrichtswissenschaft, 46, 499-522.

Helmke, A. (2010). Unterrichtsqualität und Lehrerprofessionalität. Diagnose, Evaluation und Verbesserung des Unterrichts (3. Aufl.). Seelze-Velber: Kallmeyer.

Herrmann, C., \& Gerlach, E. (2020). Unterrichtsqualität im Fach Sport - Ein Überblicksbeitrag zum Forschungsstand in Theorie und Empirie. Unterrichtswissenschaft, 48(3), 361-384.

Hossner, E.-J., Müller, H., \& Voelcker-Rehage, C. (2013). Koordination sportlicher Bewegungen: Sportmotorik. In A. Güllich \& M. Krüger (Hrsg.), Sport: das Lehrbuch für das Sportstudium (S. 211-267). Berlin: Springer.

Klieme, E. (2006). Empirische Unterrichtsforschung: aktuelle Entwicklungen, theoretische Grundlagen und fachspezifische Befunde. Einführung in den Thementeil. Zeitschrift für Pädagogik, 52(6), $765-773$.

Kurz, D. (2008). Der Auftrag des Schulsports. Sportunterricht, 57(7), 1-8.

Laging, R. (2015). Bewegungsaufgaben - ein Ansatz zur bildungs- und professionstheoretischen Aufgabenkultur im Sportunterricht. In J. Bietz, R. Laging \& M. Pott-Klindworth (Hrsg.), Didaktische Grundlagen des Lehrens und Lernens von Bewegungen - bewegungswissenschaftliche und sportpädagogische Bezüge (S. 135-159). Baltmannsweiler: Schneider Verlag Hohengehren.

Lewis, A., \& Smith, D. (1993). Defining higher order thinking. Theory Into Practice, 32(3), 131-137.

Mitchell, S., Oslin, J., \& Griffin, L. (2013). Teaching sport concepts and skills: a tactical games approach for ages 7 to 18 (3. Aufl.). Champaign: Human Kinetics.

Pfitzner, M. (2018). Lernaufgaben im kompetenzförderlichen Sportunterricht. Theoretische Grundlagen und empirische Befunde. Wiesbaden: Springer VS.

van de Pol, J., Volman, M., \& Beishuizen, J. (2010). Scaffolding in teacher-student interaction: a decade of research. Educational Psychology Review, 22(3), 271-296.

Praetorius, A. K., \& Charalambous, C. Y. (2018). Classroom observation frameworks for studying instructional quality: looking back and looking forward. ZDM, 50(3), 535-553.

Praetorius, A. K., Herrmann, C., Gerlach, E., Zülsdorf-Kersting, M., Heinitz, B., \& Nehring, A. (2020). Unterrichtsqualität in den Fachdidaktiken im deutschsprachigen Raum -zwischen Generik und Fachspezifik. Unterrichtswissenschaft, 48(3), 409-446.

Raab, M. (2007). Think SMART, not hard-a review of teaching decision making in sport from an ecological rationality perspective. Physical Education and Sport Pedagogy, 12(1), 1-22. 
Raab, M., Masters, R. S., Maxwell, J., Arnold, A., Schlapkohl, N., \& Poolton, J. (2009). Discovery learning in sports: implicit or explicit processes? International Journal of Sport and Exercise Psychology, 7(4), 413-430.

Richartz, A., \& Zoller, R. (2011). Die Qualität von Unterrichtsprozessen erfassen. In H. Lange, G. Duttler, T. Leffler, A. Siebe \& M. Zimlich (Hrsg.), Bewegungsbezogene Bildungskonzeptionen. Zur Trias Konzeption, Implementation und Evaluation. Jahrbuch Bewegungs- und Sportpädagogik in Theorie und Forschung. (S. 75-87). Baltmannsweiler: Schneider Verlag Hohengehren.

Roth, K., \& Kröger, C. (2015). Ballschule: ein ABC für Spielanfänger (5. Aufl.). Schorndorf: Hofmann.

Scherer, H. G., \& Bietz, J. (2013). Lehren und Lernen von Bewegungen. Baltmannsweiler: Schneider Verlag Hohengehren.

Scherler, K. (2006). Sportwissenschaft und Schulsport: Trends und Orientierungen (2). Sportdidaktik. Sportunterricht, 55(10), 291-297.

Stürmer, K., \& Fauth, B. (2019). Kognitive Aktivierung als zentrales Thema der empirischen Unterrichtsforschung. In A. Gawatz \& K. Stürmer (Hrsg.), Kognitive Aktivierung im Unterricht. Befunde der Bildungsforschung und fachspezifische Zugänge (S. 8-25). Braunschweig: Westermann.

Wolpert, \& Landy (2012). Motor control is decision-making. Current opinion in neurobiology, 22(6), 996-1003.

Wulf, G. (2013). Attentional focus and motor learning: a review of 15 years. International Review of Sport and Exercise Psychology, 6(1), 77-104. 